\title{
Desafíos de la colaboración digital entre museos etnológicos y comunidades indígenas: dos perspectivas, una conclusión
}

Challenges of digital collaboration between museums and indigenous communities: two perspectives, one conclusion Les défis de la collaboration numérique entre les musées et les communautés autochtones : deux perspectives, une conclusion

\section{Andrea Scholz y Mirĩgõ-Diana Guzmán Ocampo}

\section{OpenEdition}

Journals

Edición electrónica

URL: https://journals.openedition.org/jsa/19610

DOI: $10.4000 /$ jsa. 19610

ISSN: $1957-7842$

Editor

Société des américanistes

Edición impresa

Fecha de publicación: 30 septiembre 2021

Paginación: 187-204

ISSN: 0037-9174

Referencia electrónica

Andrea Scholz y Mirĩgõ-Diana Guzmán Ocampo, «Desafíos de la colaboración digital entre museos etnológicos y comunidades indígenas: dos perspectivas, una conclusión», Journal de la Société des américanistes [En línea], 107-1 | 2021, Publicado el 30 septiembre 2021, consultado el 04 septiembre 2022. URL: http://journals. openedition.org/jsa/19610 ; DOI: https://doi.org/10.4000/jsa. 19610

Este documento fue generado automáticamente el 4 septiembre 2022.

All rights reserved 


\title{
Desafíos de la colaboración digital entre museos etnológicos y comunidades indígenas: dos perspectivas, una conclusión
}

\author{
Challenges of digital collaboration between museums and indigenous \\ communities: two perspectives, one conclusion \\ Les défis de la collaboration numérique entre les musées et les communautés \\ autochtones: deux perspectives, une conclusion
}

Andrea Scholz y Mirĩgõ-Diana Guzmán Ocampo

1 Una tarea importante de los museos etnológicos hoy en día es abrir y activar sus colecciones para la colaboración con las llamadas sociedades de origen. Para los museos, estas actividades forman parte de su descolonización, lo que generalmente implica una preocupación crítica y autoreflexiva con su propia historia institucional, con un foco especial en la época colonial ${ }^{1}$. Además de esto, se discuten las prácticas de representación, renegociando la autoridad interpretativa sobre los objetos, principalmente en las exposiciones, pero también en otras prácticas museísticas, como por ejemplo la investigación de las procedencias de los objetos y los modos de conservación y de restauración ${ }^{2}$. De acuerdo con el estado actual de la discusión, todas estas actividades deben no solo respetar, sino activamente incluir las perspectivas de las sociedades de origen sobre su propio patrimonio cultural.

2 Para las comunidades, en cambio, la descolonización de los museos es generalmente un aspecto menos interesante en los proyectos de cooperación. Lo que les preocupa es el acceso a su patrimonio cultural histórico, que en muchos casos ya no existe a escala local. Por medio de colaboraciones los objetos pueden ser incluidos en procesos de revitalización y de educación propia. En otras palabras, los procesos locales, iniciados por los encuentros con las colecciones en los museos, son lo más relevante para las comunidades (véase Golding y Modest 2013; Peers y Brown 2003; Watson 2007). 
Un tema que, por lo menos implícitamente, forma parte de la colaboración entre museos y comunidades es la restitución de objetos. Por lo general, la actitud de los museos frente a esta temática no es muy abierta; sin embargo, se puede observar un cierto avance en la dinámica de discusión en los últimos años (Sarr y Savoy 2018). Hasta ahora, los museos se han mostrado muy reservados con respecto a la restitución de los objetos físicos, pero cada vez hay más proyectos en los que se restituyen "digitalmente" colecciones.

En teoría, el mundo digital abre muchas posibilidades de comunicación para ambos lados, museos y comunidades, ya que permite la articulación permanente entre mundos distantes. Sin embargo, nos parece engañoso hablar de restitución cuando los objetos físicos digitalmente compartidos permanecen en los acervos de los museos (véase también Boast y Enote 2013). Entretanto, la cooperación digital tiene el potencial de convertir las colecciones en un patrimonio cultural permanentemente compartido. En la práctica, esta comunicación entre mundos distantes no es tan fácil. A continuación, presentaremos una plataforma de colaboración digital que fue iniciada por el Museo Etnológico de Berlín en el marco del proyecto "Compartir Saberes" (2015-2021). Su objetivo fue crear accesos a la colección de la Amazonía y establecer lazos permanentes con colaboradores indígenas ${ }^{3}$ que están comprometidos en iniciativas de educación propia. La plataforma desarrollada por el proyecto entró en función en 2015. Desde finales de 2020 se encuentra fuera de línea4 ${ }^{4}$. En este texto nos enfocaremos en las experiencias de colaboración digital entre el museo y un socio específico, la Escuela Normal Superior Indígena María Reina (ENOSIMAR) en Mitú (Vaupés, Colombia), que forma jóvenes indígenas como profesores de educación primaria y alberga además un pequeño museo comunitario.

5 Este articulo inicia presentando el tema, el marco analítico y las ideas básicas de la plataforma digital. Siguen dos secciones escritas desde las diferentes perspectivas de cada una de las dos autoras. Mientras Andrea Scholz expone su punto de vista como iniciadora de la cooperación, Diana Guzmán describe sus experiencias como colaboradora del proyecto desde la localidad de Mitú. En la tensión entre las dos perspectivas, se manifiestan los retos a los que se enfrenta la colaboración digital entre museos y comunidades indígenas, que son tanto técnicos como epistemológicos, y también éticos.

\section{Colaboración digital y la inercia de las infraestructuras}

6 Para analizar el caso sistemáticamente, aplicamos un enfoque teórico feminista en "Science and Technology Studies" (STS), ya que autores como Donna Haraway y Susan Leigh Star han contribuido durante mucho tiempo tanto a cuestionar la objetividad en las ciencias como a señalar el carácter situado de todo el conocimiento y de sus infraestructuras (Haraway 1988; Leigh Star y Bowker 1999; Leigh Star y Ruhleder 1996; Bowker et al. 2015). Por infraestructura, entendemos la interacción de conocimientos, herramientas, prácticas y condiciones físicas compartidas por un grupo social. Por lo general, la infraestructura no se percibe conscientemente, sino que constituye el trasfondo invisible de tareas y de acciones concretas. De esta manera, forma parte de la organización social. Por lo general, solo nos damos cuenta de la infraestructura cuando está fallando. Por naturaleza las infraestructuras se caracterizan por su inercia inherente (Leigh Star 1999, p. 380-382). 
7 Teniendo en cuenta la historia de los museos etnológicos y de sus colecciones, estrechamente ligadas a la expansión europea y a ideas de superioridad, se puede suponer que las infraestructuras museísticas reflejan y reproducen órdenes hegemónicos de conocimiento (ver Barringer y Flynn 1998; O'Hanlon y Welsch 2000; Pearce 1999; Stocking 1985, 1991; Zimmerman 2001). Las bases de datos se relacionan directamente con las infraestructuras de estas instituciones: en ellas se crean y afirman ciertos sistemas de clasificar los objetos en las colecciones.

Utilizando el ejemplo de la clasificación internacional normalizada de la Organización Mundial para la Salud para enfermedades (International Catalogue of Diseases, ICD), Leigh Star y Bowker (1999) analizan la supuesta universalidad y, al mismo tiempo, los mecanismos habituales de los sistemas de clasificación cuya naturaleza excluye las perspectivas "marginales". En el caso del ICD, el conocimiento hegemónico de los médicos es crucial: las enfermeras y parteras, por ejemplo, o sistemas no occidentales de salud están subrepresentados. El mismo fenómeno es muy evidente en las bases de datos estándar de los museos y en los catálogos en línea. En estas bases, la perspectiva de las sociedades de origen con respecto a los objetos está mínimamente presente y francamente subrepresentada. Los coleccionistas solían obtener su información inicial de informantes locales, pero en las representaciones internas y públicas la "voz indígena" queda simplificada y es casi invisible. Las infraestructuras coloniales para clasificar y disciplinar objetos "etnográficos" se ven así confirmadas tanto en su inercia inherente como en sus mecanismos de exclusión.

9 La exclusión no solo opera mediante clasificaciones, sino también a través del diseño. Leigh Star (1999, p. 389) señala que las tecnologías suelen estar diseñadas para funcionar en una infraestructura, pero no en otra. Uno de sus ejemplos más llamativos para esto son los puentes de acceso para Long Island (Nueva York), que fueron construidos a altura baja para que los autobuses del transporte público no pudieran entrar. En vez de prohibir explícitamente el acceso a la isla a la gente de bajos recursos, esta población se vio excluida mediante la planificación urbana. Lo que se expresa en este ejemplo es una infraestructura de ricos. Aplicando el análisis de estos mecanismos a nuestro ejemplo de los museos etnológicos, veremos que las exclusiones se manifiestan de muchas maneras, también en aquellos proyectos que se comprometen a tomar caminos alternativos, descoloniales. A continuación, revelaremos algunos de estos obstáculos en la descolonización de los museos, centrándonos en el trabajo invisible de los sistemas de comunicación.

\section{Experiencia en cuatro años de colaboración digital}

Con la plataforma "Compartir Saberes" el museo pretendió enriquecer las informaciones acerca del significado y origen de los objetos en la colección, así como cuestionar y renegociar las formas actuales de clasificación, de conservación y de representación del museo (Scholz 2018). Tras un proyecto piloto en el marco del Humboldt Lab Dahlem (2014-2015) $)^{5}$, el proyecto ha sido financiado desde 2016 por la Fundación Volkswagen en el marco del programa "Investigación en Museos" bajo el nombre "Cosas vivas en la Amazonía y en el Museo-Saberes Compartidos en el Humboldt Forum". La meta del proyecto era llevar a cabo investigaciones colaborativas sobre la colección, utilizando una plataforma web que facilitaría la comunicación y el almacenamiento de los datos generados. Con las informaciones más complejas se pretendían alimentar también las 
instalaciones interactivas en la exposición planificada para el Humboldt Forum ${ }^{6}$, con el propósito de representar en forma directa las perspectivas indígenas.

Hasta el año 2016, el único socio fue la Universidad Experimental Indígena del Tauca (UNEIT) en Venezuela y el enfoque estuvo en las colecciones de los Pemón y Ye'kwana. Con la nueva financiación, se integraron socios de Brasil y Colombia y se añadieron objetos de la región del Alto Río Negro (Brasil/Colombia). Algunos de los socios, como por ejemplo ENOSIMAR, tienen sus propias colecciones, que también se compartieron a través de la plataforma. Un total de 1500 objetos estaba accesible en línea hasta muy recientemente.

La plataforma se basó en MongoDB, una base de datos NoSQL que fue creada para manejar documentos. Cada documento reunía información sobre un objeto etnográfico. Lo excepcional de "Compartir Saberes" era que todas las informaciones sobre los objetos podían ser editadas y anotadas, lo que en su totalidad constituyó la investigación colaborativa. A diferencia de las bases de datos relacionales convencionales, como por ejemplo "Museum Plus", que se usa internamente en el museo, "Compartir Saberes" almacenaba la historia de edición de un objeto, y las y los usuarias/os tuvieron que justificar sus entradas. De esta manera, todos los cambios permanecieron rastreables. La investigación acerca de los objetos se entendió como un proceso dinámico, nunca terminado.

El concepto de la plataforma fue desarrollado en 2014, durante la primera visita de una delegación de la UNEIT a Berlín. Al trabajar con los objetos en el depósito, quedó claro que el modo de clasificación en la base de datos interna, cuya referencia principal son los coleccionistas y los términos genéricos, no tenía mucho sentido para los socios indígenas. En cambio, ellos se orientaron principalmente hacia el contexto cultural de origen de los objetos. Conforme con esta concepción, la página de inicio mostraba un mapa dibujado de la región de la Amazonía con símbolos que hacían referencia a grupos étnicos o, en el caso del Alto Río Negro, a un patrimonio cultural compartido entre varios grupos (Figura 1). 
Fig. 1 - Página de inicio de la plataforma digital

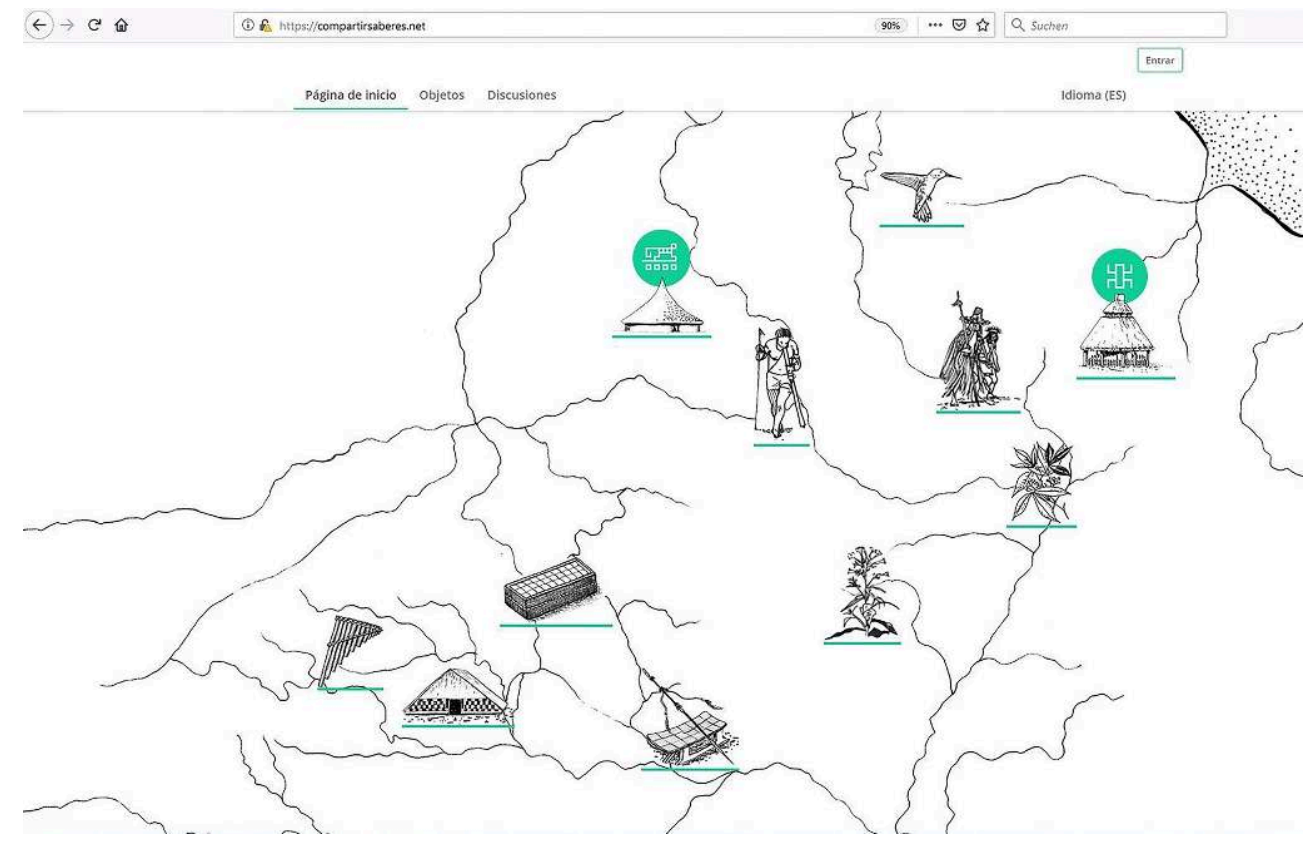

(gráfica y concepto: Studio NAND y Museo Etnológico de Berlín)

Además de los rasgos geográficos de orientación, el mapa contenía también símbolos que servían para unir objetos relacionados con ciertos contextos rituales. Técnicamente, estos símbolos estaban vinculados a términos de búsqueda simples, lo que limitaba en gran medida las posibilidades de vinculación lógica de entidades y, por lo tanto, redujo la complejidad de conexiones entre objetos. Una de las principales debilidades técnicas y a su vez epistemológicas de la plataforma residía en ese reduccionismo.

La decisión de trabajar con símbolos en lugar de texto fue una consecuencia de la naturaleza multilingüe del proyecto. Además de en español y en portugués, los idiomas más importantes para la comunicación entre todos, la plataforma permitía hacer ediciones en alemán, inglés y en ocho idiomas indígenas. La plataforma y sus subpáginas individuales se repitieron en todos estos idiomas, pero la interfaz del usuario solo fue traducida de forma incompleta debido a la falta de recursos humanos $\mathrm{y}$, en última instancia, financieros.

La función de búsqueda en la página de inicio conducía a una lista de objetos filtrados temáticamente con fotos de previsión. Cada ítem en la lista estaba vinculado a la página individual de un objeto (Figura 2). 
Fig. 2 - Vista de una Cesta Ye'kwana en la plataforma

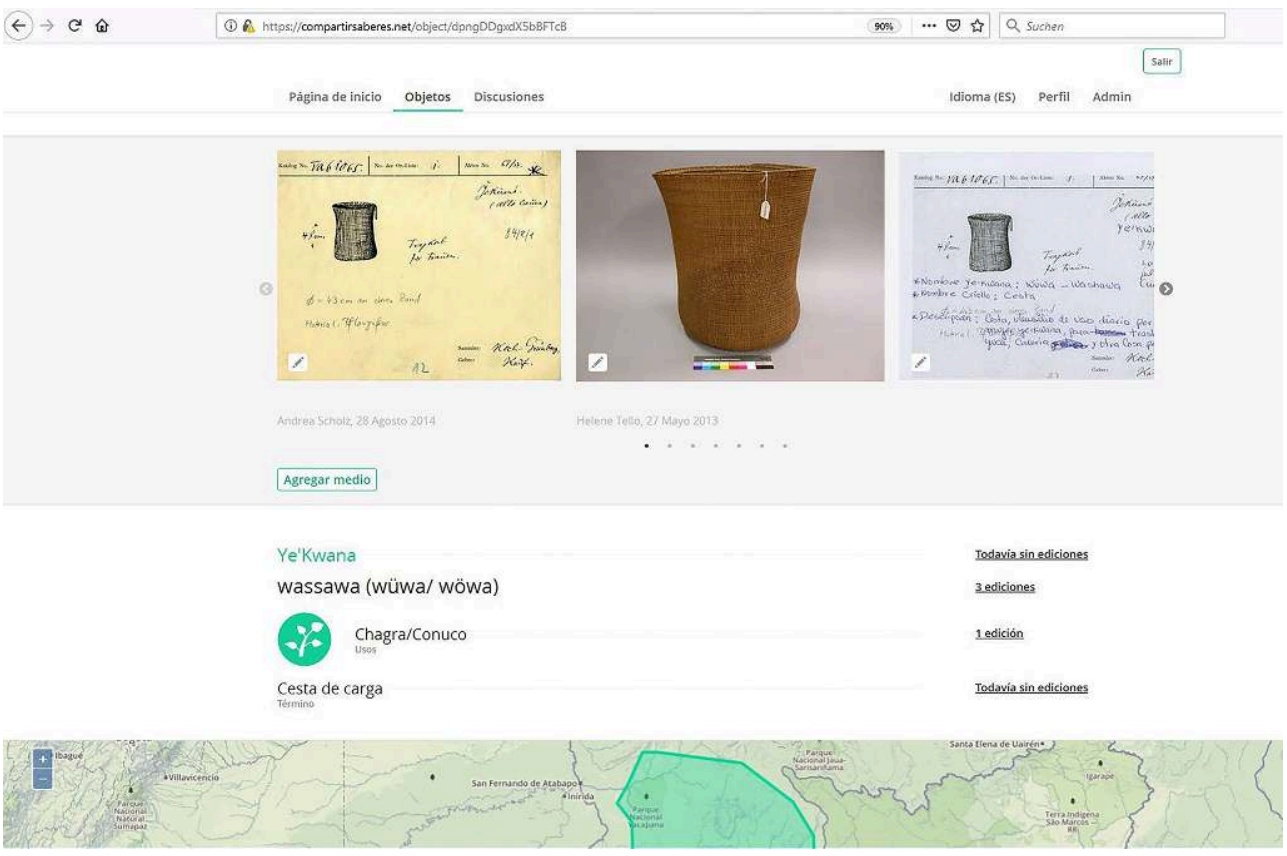

(gráfica y concepto: Studio NAND y Museo Etnológico de Berlín)

La presentación de la información se basaba en una jerarquía definida conjuntamente con los socios de la UNEIT, que en muchos aspectos resultó ser exactamente lo contrario de la clasificación del museo. La primera y más importante fue la clasificación "étnica", que en la base de datos interna del museo desaparece bajo las "referencias geográficas". A continuación, se designaba el objeto por un nombre en la lengua indígena respectiva, un símbolo que transmitía el contexto de uso (no disponible en la base de datos interna) y el término genérico de la clasificación usual en el museo. En el centro de la página del objeto había una herramienta cartográfica que permitía vincular el objeto con una información histórico-geográfica (por ejemplo, lugar de manufactura o colección; región en la que se produce y/o utiliza el objeto en la actualidad; lugar donde se guarda el objeto).

Además de textos, se podían integrar medios audiovisuales (fotografías o grabaciones en video o audio) a la página del objeto. El museo proporcionaba por lo menos una foto del objeto y el escaneado de la ficha histórica. Las usuarias y los usuarios podían subir aquí más fotos, archivos de audio y de vídeo. Esta función se ubicaba en la parte superior de la página, ya que los socios indígenas enfatizaron desde el principio que era importante expresar la "vida de las cosas". Con esto se refirieron a la producción y al uso de las cosas en sus comunidades. Este deseo de recontextualizar los objetos apunta a una contradicción muy profunda entre las infraestructuras del conocimiento (museo versus mundo indígena), mencionada también por Fabian (1991, p. 205): "The emergence of Culture as a guiding concept in anthropology signaled the victory of representationalism. [...] As a dominant idea in the Western tradition, it was made to serve the project of domination of other peoples by the West [...]"' 


\section{Infraestructuras en conflicto - un análisis desde el punto de vista de la iniciadora (Andrea Scholz)}

En el museo, los objetos etnográficos se negocian como representaciones del conocimiento, mientras que los mismos objetos, a pesar de estar musealizados, para los socios indígenas forman parte de un conocimiento incorporado que no puede ser reducido en su complejidad a una representación externalizada de las prácticas (SantosGranero 2009, p. 2). Dentro del marco del trabajo con la plataforma, la solución consistió en integrar, siempre que fuera posible, el aspecto práctico en forma de grabaciones de vídeo y audio. Aun así las posibilidades de articulación entre las infraestructuras de conocimiento seguían siendo limitadas, ya que la información de la plataforma se centraba en el artefacto. Para los socios indígenas, sin embargo, toda una serie de conexiones lógicas adicionales son relevantes, incluyendo las materias primas, que a su vez establecen conexiones con el territorio. Los mitos, en los que todos estos aspectos se unen, forman un nivel más de significado o, en muchos casos, un marco superior de integración (véase Hugh-Jones 2009, p. 35-41).

Esta percepción llevó a que el aspecto digital retrocediera al segundo plano en el proyecto. En lugar de describir objetos en línea, se planearon talleres centrados en la producción de objetos. En el caso de ENOSIMAR, los talleres fueron llevados a cabo tanto en comunidades Kotiria y Cubeo como también en el colegio. El taller que se organizó en marzo de 2018 en la comunidad indígena de Macucu (del pueblo Kotiria) se centró en la elaboración de artefactos de diferentes fibras vegetales (cumare, bejuco, arumá) (Figura 3).

Fig. 3 - Taller de fibras en Macucu

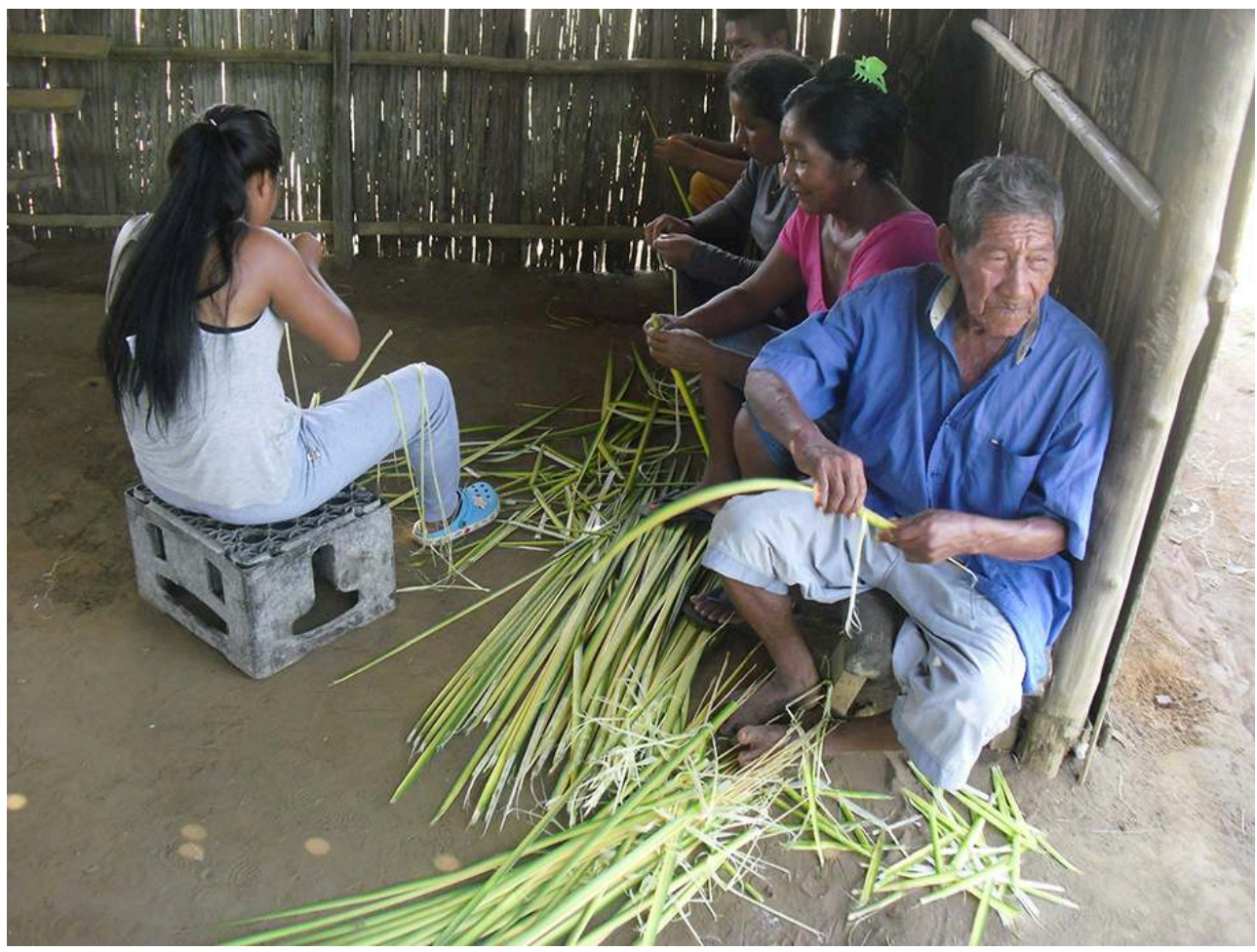

(foto: Orlando Villegas, 2018) 

ancianos contaban historias sobre el origen de las plantas y de los objetos. Las actividades fueron filmadas con el objetivo de documentar el proceso y de enriquecer la colaboración digital con los resultados.

Sin embargo, en la arquitectura de datos de la plataforma antes descrita no era posible vincular entre sí las diferentes entidades lógicas (artefacto, material, territorio, origen). Debido a la concentración en el objeto (= documento en MongoDB), se trataba cada información adicional como atributo que no puede tener conexiones con otros atributos (por ejemplo, el material con el origen). Contrariamente a la intención original de modificar las formas de clasificación, la infraestructura del museo siguió apropiándose del conocimiento indígena. Se trataba de un "defecto de nacimiento" de la plataforma "Compartir Saberes". En lugar de alinear la arquitectura de datos con la complejidad de dos infraestructuras diferentes, el punto de partida fue la exportación de información de la base de datos interna que refleja la infraestructura del museo. Aun si la información en la plataforma expresaba una jerarquía diferente y podía ser editada más tarde, el sistema básico de clasificación permaneció así inalterado. Otro problema era que la plataforma nunca dejó de ser resultado de un proyecto temporal y externo. Las exportaciones solo se realizaban desde la base de datos interna a la plataforma, la información no fluía en el otro sentido. El sistema interno permanecía inmutable. Por último, el hecho de que la plataforma se haya apagado tras la finalización del proyecto también fue una consecuencia de su carácter temporal y, sin un proyecto de seguimiento, la información recopilada se hubiera perdido irremediablemente.

Además de las debilidades conceptuales y estructurales, la plataforma tenía otro defecto de nacimiento fatal, que fue particularmente evidente en la cooperación con ENOSIMAR: la falta de un modo offline practicable. Por el acceso limitado a Internet en Mitú, los estudiantes consultaban una exportación fuera de línea de la plataforma que era muy simplificada, ya que consistía en una lista vinculada a archivos en HTML. No incluía la posibilidad de realizar búsquedas o de editar la información, así que los estudiantes introdujeron sus propias observaciones en formularios de papel. Sin embargo, la posibilidad de describir libremente los objetos en los formularios produjo resultados que, una vez más, expresaron claramente las diferencias entre las infraestructuras de conocimiento y los déficits de la plataforma.

En el ejemplo ilustrado en la Figura 4, María Idaly Mejía eligió una pieza de cerámica para la descripción, que fue recogida por el coleccionista Theodor Koch-Grünberg en Brasil, entre los Baniwa, un grupo Arawak que habita hasta hoy en día en la región de los ríos Isana e Ayarí. Esta información se podía encontrar en la plataforma. María Idaly Mejía pertenece al grupo Kotiria del Bajo Río Vaupés, pero creció en la comunidad Barasana de su padrastro en la cuenca del Pira-Paraná (Colombia). Por conocer el objeto del lugar donde fue criada, ella designó a los Barasana como "sociedad de origen", entre otros grupos también representados en el Pira-Paraná. Su descripción comienza con el origen del material (arcilla) que proviene del cuerpo de un espíritu maligno llamado wãtĩ gtule magĩ. En el mismo formulario explica que Menicumu, el primer hombre, mató al espíritu después de que este había matado a sus hijos. Algún tiempo después la esposa de Meni, Yaia Bnasagõ, fue al cadáver del espíritu para mirarlo. Su marido le explicó en voz alta que este cuerpo no se perdería, sino que se convertiría en un material con el que se podrían fabricar importantes utensilios para el hogar y los rituales. Según María Idaly Mejía, la arcilla, cuyo origen se deriva de los restos del espíritu maligno, se encuentra en 
lugares especiales, sobre todo en pequeños afluentes. Para procesarla, se mezcla con otros materiales. La cerámica es usada como plato para comer pescado u otros alimentos.

Fig. 4 - Planilla para la descripción de una cerámica redactada en marzo de 2018 en Mitú (Vaupés, Colombia) por María Idaly Mejía Montalvo (grupo Kotiria, estudiante de ENOSIMAR en este momento)

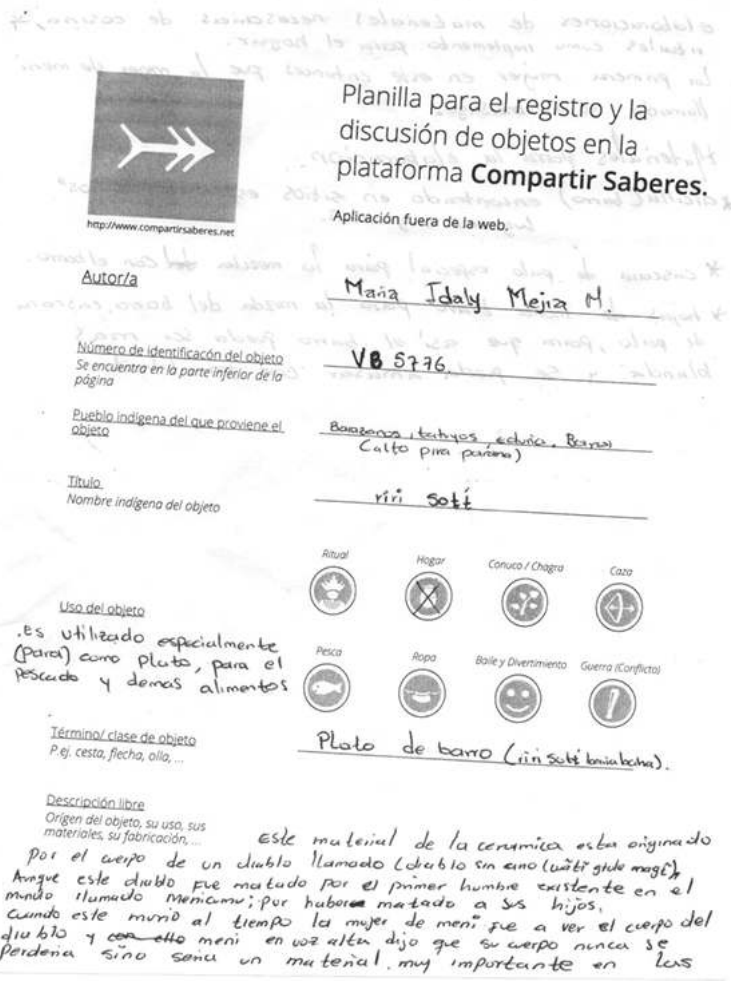

Este ejemplo muestra con claridad que los estudiantes de ENOSIMAR percibieron los objetos del Alto Río Negro guardados en el museo etnológico de Berlín como propiedad común de los grupos de la región. El lugar concreto y el contexto de adquisición no tenían mucha relevancia. Aunque la plataforma les permitía ver con qué grupo indígena y en qué región fue recolectado, ellas y ellos describieron los objetos sobre la base de su propia afiliación étnica y de sus prácticas y actividades en su comunidad. Las descripciones se centraban, por un lado, en las técnicas de producción y uso, por otro, en el origen mítico que conecta el objeto con el territorio. Debido a las alianzas matrimoniales y a las estrechas relaciones entre grupos lingüísticamente distintos que viven en diferentes territorios, la mayoría de los objetos que se encuentran en la colección de Berlín son generalmente conocidos por diferentes grupos, y todavía existen en la región en la actualidad. Los mitos de origen difieren de un lugar a otro; forman una red compleja y algunas veces contradictoria de relatos que difícilmente pueden ser simplificados. Por el contrario, los temas centrales para la investigación occidental, como lo son las procedencias, el productor de un objeto concreto y su cambio de propiedad, resultaron de poco interés. El coleccionista, tan importante desde el punto de vista europeo, también se convirtió en una figura marginal, como ya habíamos visto en el caso de los estudiantes de la UNEIT.

La riqueza de información interconectada, expresada en la planilla de papel, simplemente no podía ser desplegada en la "página del objeto" de la plataforma. Los resultados refuerzan una vez más la imposibilidad de vincular información compleja dentro de la 
propia plataforma. Sin embargo, el trabajo con las planillas resultó poco práctico y poco atractivo para los estudiantes. El intento posterior de implementar una solución offline puede ser visto como revelador de un fracaso inicial. Se parece al ejemplo del puente de Long Island en Nueva York, que no deja pasar a los autobuses.

\section{Infraestructuras en conflicto -un análisis desde Mitú (Mirĩgõ-Diana Guzmán Ocampo)} intentar abrir espacio para escuchar las voces de los pueblos indígenas y lo que pueden aportar frente a las "colecciones" que reposan en los museos. Viendo la estrategia desde nuestra realidad geográfica e histórica, es algo llamativo porque es dar a conocer lo nuestro y presentarlo de una forma moderna en la que la tecnología juega un papel importante para las comunicaciones. Pero desde nuestra realidad estos intentos, así como han generado muchas expectativas para nosotros mismos, también se han convertido en un pequeño dolor de cabeza, ya que no poseemos los medios tecnológicos suficientes para cumplir con las expectativas propuestas.

Hablando de la experiencia como tal, el entrar a la plataforma, los pasos a seguir, las puertas por abrir fueron muchas y muy diversas. Con la baja conectividad a Internet que se tiene en el territorio del Vaupés, y especialmente en el casco urbano que es la única parte donde se cuenta con señal, el trabajo se complicó mucho debido a que se demoraba bastante en descargar y, cuando se estaba redactando algún texto, la señal fallaba y todo lo realizado en ese momento se perdía. Esta fue una de las dificultades. Como hemos visto anteriormente, habíamos optado por hacer uso de las planillas, y reenviarlas por medio del teléfono móvil, pero también encontramos allí un obstáculo frente a la capacidad que se tiene o se requiere para el envío de archivos, ya que hay documentos que desafortunadamente no pueden enviarse por este medio, quedando así nuevamente mucha información guardada/incomunicada, como es el caso de los videos o grabaciones en general.

Ahora, nuestra forma de clasificar el conocimiento difiere bastante de las estructuras planteadas por los museos. Empezando por el mismo término museo, que hace referencia a algo que ya desapareció, a unas sociedades que ya murieron y de las cuales solo quedan "objetos". En el sistema de pensamiento cultural indígena todo se conecta, cada cosa forma parte de un todo. Para nosotros, la importancia no radica en el lugar donde encontraron el objeto, sino en lo que representa y en la historia que narra de un pueblo, porque los objetos como la gente circulan. Las informaciones recolectadas por los coleccionistas para nosotros están vacías, solas, aisladas y sin embargo esta información es la que luego va a difundirse en los medios académicos y científicos. Vemos con preocupación cómo se crean conceptos, definiciones y explicaciones sobre ellas con lo poco que se tiene. Por eso, encontramos en la estrategia de colaborar directamente con los grupos indígenas una manera de complementar de viva voz la existencia de ese ser al cual se llama "colección", reencontrarlo y reconectarlo con sus otras partes. Las colecciones contienen muchos elementos de nuestra cultura (por ejemplo, ornamentos de danza), que se mantienen lejos de nosotros, fuera de contexto, alejados unos de los otros en las bodegas y que, la mayoría de las veces, son incomprendidos. Creemos que es fundamental encontrar la manera de reestablecer el lazo de los elementos culturales con el territorio, con la vida misma y, especialmente, con nosotros.

Journal de la Société des américanistes, 107-1 | 202 
31 Este proceso es de particular relevancia pensando en las nuevas generaciones y en nuestro compromiso como docentes y como formadores de docentes para el territorio. Se habla con los jóvenes maestros que forman parte del proyecto de la importancia de dar a conocer a las nuevas generaciones los aportes positivos que nos puede prestar la tecnología. Esta actividad es vista como una herramienta que nos sirve para reunir, guardar las imágenes y voces de nuestros abuelos, de nuestras prácticas culturales propias. Estas nos permitirán alimentar y recordar aspectos muy importantes de nuestro patrimonio e identidad, compartirlos con nuestros niños, niñas y jóvenes, convirtiéndose así en una ayuda para la preservación y conservación de la cultura en el tiempo. A las nuevas generaciones, les llama poderosamente la atención ver unidas la imagen, la acción y el sonido en una sola presentación como por ejemplo un video, porque le da más cercanía y vida al objeto que allí se expone.

Estamos buscando romper con el paradigma de que solo se aprende en la escuela. Los niños, niñas y jóvenes tienen también conocimientos, construyen conocimiento a partir de sus saberes propios. Esta es una forma de hacer que el conocimiento circule, pues lo que se ve en la escuela vincula a los padres de familia y, a su vez, a las diferentes comunidades y grupos étnicos a los cuales pertenecen los maestros, los estudiantes y los padres de familia. La meta es crear los vínculos y entrelazarlos con todo el territorio. Es este el ideal que hemos fomentado internamente en la ENOSIMAR: participar activamente y compartir los saberes para tejer el conocimiento. Pero cuando regresamos a la realidad, el hecho de hacer y de compartir queda reducido a una mínima parte.

Uno de los objetivos de la plataforma era precisamente permitir que el saber indígena pudiera expresarse, conectarse y tratar de explicar la esencia de las mismas cosas, abrir el espacio para poder aclarar, dilucidar las líneas que unen los objetos con el territorio y la importancia de este en las sociedades indígenas y en la actualidad. Entendiendo el territorio no solo como un espacio geográfico con límites y fronteras, sino como el espacio donde se crea, se nace, aprende, crece, comparte, habla, vive y retorna a los orígenes. Pero, nuevamente, por razones técnicas y de infraestructura estas puertas en la plataforma no se conectaban, estaban allí, pero seguían aisladas unas de otras.

Para nosotros, es importante dar a conocer a los abuelos lo que se está haciendo, llevar la palabra y la acción de lo hecho a los territorios. Pero es allí también donde encontramos dificultades porque solo podemos presentarlo por medio de imágenes nuevamente sueltas, aisladas de todo un proceso.

La explicación se nos vuelve complicada y fría. Y dentro de nuestras formas propias de enseñanza y aprendizaje lo hacemos haciendo: es decir que para poder entender hay que hacer, actuar, participar, no tener miedo de equivocarnos y volver a empezar. Desafortunadamente, aun con las dinámicas propias de la plataforma, esto nos ha quedado difícil.

Pero lo dicho no significa que todo haya sido malo, o que no haya servido. Todo lo contrario. Frente a estas dificultades expuestas, es que hay que actuar, seguir buscando estrategias que permitan realmente que la voz de la selva se escuche, que se le preste atención y que se convierta en un aliado para la construcción del conocimiento. Que los aportes nuestros sean bien recibidos, no como informantes sino como depositarios y dueños del conocimiento de nuestro patrimonio, el cual se quiere compartir sin egoísmos para fortalecerlo día a día, haciendo una buena comprensión y buen uso del desarrollo tecnológico y de todo lo que nos puede aportar la ciencia. 


\section{Discusión, conclusión y perspectivas}

En resumen, el proyecto "Compartir Saberes" fue un experimento bien intencionado de abrir el museo a perspectivas de las llamadas sociedades de origen. Se creó una plataforma web que permitió la expresión de saberes indígenas pero que, al mismo tiempo, quedó limitada en muchos aspectos. Hasta un cierto punto, esto puede ser visto como normal, puesto que "todo conocimiento es local" (Bowker 2018). Desde el punto de vista del museo, el proyecto forma parte de un discurso de culpa histórica, colonial, en el que la institución como sistema de conocimiento local se refleja a sí misma, pero no escapa a sus lógicas y, por tanto, a sus órdenes de conocimiento. Por otro lado, el conocimiento indígena, como hemos visto también, se expresó más fuertemente en los talleres, donde reanudaba con la práctica de lidiar con los llamados objetos. Los mecanismos de exclusión se manifestaron sobre todo en el desconocimiento, por parte del museo, de las condiciones locales (acceso deficiente a Internet) y en la creación de una arquitectura de datos que, al contrario de la motivación inicial, reflejaba los patrones de clasificación habituales del museo.

¿Cómo podrían solucionarse los problemas que acabamos de analizar y que, en última instancia, parecen impedir una buena comunicación entre museos y comunidades indígenas por vía digital?

39 En primer lugar, la cooperación sostenible entre museos y las llamadas sociedades de origen tendría que reflejarse a largo plazo en una institucionalización de los formatos digitales, en los que el saber indígena no se externaliza a proyectos temporales, sino que se convierte en una parte integral de la producción de conocimiento, tanto en los museos como también en las escuelas y comunidades indígenas. Esta infraestructura digital permanente para la cooperación tendría que, desde el principio, adaptarse flexiblemente a las necesidades de los diferentes grupos de usuarios. En los términos de Bowker (2018), esto correspondería a una humanización de las infraestructuras. Formatos flexibles implican tecnologías inclusivas, con soluciones offline fáciles de usar y de llevar a las comunidades, además de interfaces multilingües que reflejan las infraestructuras del conocimiento de las usuarias y los usuarios, involucrándolas en los procesos creativos de su desarrollo.

Según Leigh Star y Ruhleder (1996) existe la posibilidad de articular infraestructuras complejas e incluso contradictorias, pero esta articulación requiere un trabajo de traducción no reduccionista, que insista en la "diferencia no reducible y la multiplicidad radical de conocimientos locales" (Haraway 1988, p. 579). ¿Cómo puede funcionar en la práctica? Por un lado, se necesita de personas que desempeñen un papel clave como mediadoras. Por otro lado, pensando más en los aspectos tecnológicos, nos parece interesante el concepto de los llamados objetos de frontera, descritos por Leigh Star y Griesemer (1989) en su análisis de los procesos de trabajo colaborativo en un museo de historia natural. Estos procesos fueron integrados tanto por profesionales de diferentes disciplinas como también por aficionados. Los objetos de frontera son cosas abstractas y/ o concretas, cuya flexibilidad interpretativa permite definir objetivos comunes $\mathrm{y}$, al mismo tiempo, mantener la autonomía entre los actores involucrados. Estos objetos se producen en diferentes fases de producción de conocimiento y de la comunicación y facilitan la articulación, ya que hacen referencia a sistemas diferentes de conocimiento. Leigh Star y Griesemer (1989, p. 411) comparan los objetos de frontera con personas marginales que pertenecen a diferentes mundos sociales al mismo tiempo. 
El museo etnológico como repositorio representa en sí mismo un objeto de frontera, al igual que los artefactos, mapas, notas de campo, índices de materias primas y listas de términos genéricos. Todos ellos tienen un significado y una función diferentes para los pueblos indígenas, para los coleccionistas y para los restauradores y restauradoras. Son resultado de la comunicación y permiten su continuación (ver Leigh Star y Griesemer 1989). Podemos imaginarnos que el concepto de objeto de frontera funciona como un punto de partida para repensar la colaboración digital. El desafío es captar los diferentes significados y referencias de los objetos de frontera en los mundos sociales que se cruzan dentro de ellos y entender el objeto de frontera como punto de articulación.

Por consiguiente, a mediano plazo, tenemos previsto migrar la plataforma del proyecto "Compartir Saberes" a un sistema basado en la red semántica. Mediante la definición de relaciones y lógicas, en las que una unidad digital se define por su relación con otra unidad digital ("ontologías"), la organización jerárquica del conocimiento se disuelve hasta cierto punto, ya que las definiciones de entidades y relaciones pueden provenir de diferentes infraestructuras de conocimiento. Esto permitiría una vinculación más flexible entre diferentes unidades (artefactos, materiales, recursos audiovisuales, documentos), sin tener que centrarse en el artefacto. Para adaptarse a las diferentes infraestructuras del conocimiento, el desarrollo de las "ontologías", en el sentido anterior, tendría que partir de los objetos de frontera ${ }^{8}$.

Nuestro deseo, para el futuro, es aprovechar la experiencia de los últimos cuatro años para construir un nuevo sistema que sea sostenible y apropiado para todos los que lo vayan a utilizar, incluyendo también a las personas que viven sin acceso a Internet en las comunidades indígenas y al público de futuras exposiciones y eventos, tanto en los museos como también en las comunidades. Esperamos que progresivamente puedan eliminarse los obstáculos que representan nada menos que órdenes hegemónicos de conocimiento.

\section{BIBLIOGRAFÍA}

BARRINGER Tim y Tom FLYNN (eds)

1998 Colonialism and the Object. Empire, Material Culture and the Museum (Museum Meanings), Routledge, London/New York.

BOAST Robin y Jim ENOTE

2013 "Virtual repatriation. It's virtual, but it's not repatriation", in Peter Biehl y Christoph Prescott (eds), Heritage in Context of Globalization, Europe and the Americas, Springer, New York, p. 103-113.

\section{BOWKER Geoffrey}

2018 "Sustainable knowledge infrastructures", in Nikil Anand, Akhil Gupta y Hannah Appel (eds), The Promise of Infrastructure, Duke University Press, Durham, p. 203-222. 
BOWKER Geoffrey, Stefan TIMMERMANS, Adele E. CLARKE y Ellen BALKA

2015 Boundary Objects and Beyond. Working with Susan Leigh Star, Cambridge University Press, Cambridge.

BYRNE Sarah, Anne CLARKE, Rodney HARRISON y Robin TORRENCE (eds)

2011 Unpacking the Collection. Networks of Material and Social Agency in the Museum, Springer, New York

ESCOBAR Arturo

2008 Territories of Difference. Place, Movements, Life, Redes, Duke University Press, Durham.

FABIAN Johannes

1983 Time and the Other. How Anthropology Makes Its Object, Columbia University Press, New York.

FABIAN Johannes

1991 Time and the Work of Anthropology. Critical Essays 1971-1991, Harwood, Amsterdam.

GOLDING Viv y Wayne MODEST (eds)

2013 Museums and Communities. Curators, Collection and Collaboration, Routledge, London.

\section{HARAWAY Donna}

1988 "Situated knowledges: The science question in feminism and the privilege of partial perspective", Feminist Studies, 14, p. 575-599.

HARRIS Clare y Michael o'HANLON

2013 "The future of the ethnographic Museum", Anthropology Today, 29 (1), p. 8-12.

HUGH-JONES Stephen

2009 "The fabricated body. Objects and ancestors in Northwest Amazonia", in Fernando SantosGranero (ed.), The Occult Life of Things. Native Amazonian Theories of Materiality and Personhood, University of Arizona Press, Tucson, p. 33-59.

LEIGH STAR Susan

1999 "The ethnography of infrastructure", American Behavioral Scientist, 43 (3), p. 377-391.

LEIGH STAR Susan Y James GRIESEMER

1989 'Institutional ecology, 'translations' and boundary objects: Amateurs and professionals in Berkeley's Museum of vertebrate zoology, 1907-1939”, Social Studies of Science, 19 (3), p. 387-420.

LEIGH ST AR Susan y Geoffrey BoWKER

1999 Sorting Things Out. Classifications and Its Consequences, Cambridge University Press, Cambridge.

\section{LEIGH STAR Susan y Karen RUHLEDER}

1996 "Steps towards an ecology of infrastructure: Design and access for large information spaces", Information Systems Research, 7 (1), p. 11-134.

KARP Ivan y Steven D. LAVINE

1991 Exhibiting Cultures. The Poetics and Politics of Museum Display, Smithsonian Institution Press, Washington (DC).

MCCARTHY Conal

2007 Exhibiting Māori. A History of the Colonial Culture of Display, Bloomsbury, Wellington.

\section{MCCARTHY Conal}

2011 Museums and Māori. Heritage Professionals, Indigenous Collections, Current Practice, Bloomsbury, Wellington. 
MARSTINE Janet (ed.)

2011 Routledge Companion to Museum Ethics. Redefining Ethics for the Twenty-First Century Museum, Routledge, London/New York.

O'HANLON Michael y Robert WELSCH (eds)

2000 Hunting the Gatherers. Ethnographic Collectors, Agents and Agency, Berghahn, New York/

Oxford.

PEARCE Susan

1999 On Collecting. An Investigation into Collecting in the European Tradition, Berghahn, New York/ Oxford.

PEERS Laura y Alison BRown (eds)

2003 Museums and Source Communities, Routledge, London.

SANTOS-GRANERo Fernando (ed.)

2009 The Occult Life of Things. Native Amazonian Theories of Personhood and Materiality, University of Arizona Press, Tucson.

SARR Felwine y Benedicte SAVOY

2018 The Restitution of African Cultural Heritage. Towards a New Relational Ethics, https:// restitutionreport2018.com/sarr_savoy_en.pdf, consultado el 30/06/2021.

scholz Andrea

2018 "Tejiendo nuevos enlaces: La revitalización de una colección etnográfica por la plataforma Compartir Saberes”, Mundo Amazónico, 9 (1), p. 119-142, https://revistas.unal.edu.co/index.php/ imanimundo/article/ 20view/64354/65956, consultado el 30/06/2021.

SIMPSON Moira G.

2001 Making Representations. Museums in the Post-Colonial Era, Routledge, London/New York.

STOCKING George

1985 Objects and Others. Essays on Museums and Material Culture, University of Wisconsin Press, Madison.

\section{STOCKING George}

1991 Colonial Situations. Essays on the Contextualization of Ethnographic Knowledge, University of Wisconsin Press, Madison.

\section{TUHIWAI SMITH Linda}

1999 Decolonizing Methodologies. Research and Indigenous Peoples, Zed Books, London.

VERGo Peter (ed.)

1989 The New Museology, Reaktion Books, London.

WATSON Sheila (ed.)

2007 Museums and their Communities, Routledge, London.

ZIMMERMAN Andrew

2001 Anthropology and Antihumanism in Imperial Germany, University of Chicago Press, Chicago. 


\section{NOTAS}

1. La descolonización de los museos etnológicos fue promovida decisivamente por la llamada museología nueva o crítica (véase Byrne et al. 2011; Harris y O'Hanlon 2013; Karp y Lavine 1991; Marstine 2011; Simpson 2001; Vergo 1989).

2. En cuanto a la representación de objetos etnográficos en exposiciones y publicaciones, los museos están recuperando el terreno perdido, ya que la crisis de la representación etnográfica se ha discutido en la antropología desde los años ochenta. La teoría poscolonial y las intervenciones de los llamados native scholars han aportado argumentos adicionales para la renegociación del poder interpretativo (Escobar 2008; McCarthy 2007 y 2011; Tuhiwai Smith 1999).

3. Socios indígenas en la actualidad: Instituto Socioambiental/Federação das Organizações Indígenas do Rio Negro (Brasil), Escuela Normal Superior Indígena María Reina (Colombia), Organización Indígena de la Cuenca del Caura "Kuyujani” (Venezuela), Conselho Indígena de Roraima e Associação Wanasseduume Ye'kwana (ambos de Brasil). En la fase actual del proyecto, no está claro si continuará la cooperación con la Universidad Nacional Experimental Indígena del Tauca (Venezuela).

4. La plataforma "Compartir Saberes" será almacenada y reemplazada por un sistema nuevo cuya concepción está basada en las experiencias aquí discutidas (ver nota 8).

5. Véase http://www.humboldt-lab.de/projektarchiv/index.html (consultado el 30/06/2021).

6. El Foro Humboldt es un controvertido proyecto cultural alemán que se desarrollará entre los muros de un palacio imperial reconstruido en el centro de Berlín. Abrirá sus puertas al público durante 2021 y expondrá, entre otras, las colecciones etnográficas de los museos nacionales de Berlín (https://www.humboldtforum.org/en).

7. Véase también Fabian 1983.

8. Bajo el marco de un nuevo proyecto llamado "Conectar-Comprender-Comunicar. La Amazonía como laboratorio del futuro" (2021-2023), financiado por la Fundación Alemana para la cultura, se está creando una nueva base de datos que contará con diferentes herramientas de comunicación, dependiendo del contexto de los usuarios. Las informaciones del proyecto "Compartir Saberes" serán integradas a esta nueva base. Además, en la cooperación entre el museo y ENOSIMAR, se están produciendo nuevos objetos de frontera. En otro proyecto que tiene por objetivo compartir las experiencias con la pandemia ("The Relational Museum. Sharing Knowledge in Epidemics and Crises on the upper Negro river, Brazil/Colombia", 2020-2022), se están recolectando relatos y representaciones en dibujos que ayudarán a conectar mundos que parecen cada vez más distantes.

\section{RESÚMENES}

Las plataformas digitales ofrecen muchas oportunidades para la apertura y la exploración transcultural de las colecciones de los museos. Del año 2015 al 2021, el proyecto "Compartir 
Saberes" del Museo Etnológico de Berlín se enfrentó a los retos asociados a este nuevo campo de trabajo colaborativo. La experiencia adquirida junto con los socios indígenas de América Latina muestra que la creación de nuevas infraestructuras de conocimiento requiere un replanteamiento importante. No es suficiente abrir las colecciones a las perspectivas de los demás. Una colaboración digital exitosa debe reflejar la heterogeneidad de las infraestructuras de conocimiento de los participantes.

Digital platforms offer many opportunities for the opening and transcultural exploration of museum collections. From 2015 to 2021, the project "Sharing Knowledge" at the Ethnological Museum of Berlin has been meeting the challenges associated with this new field of collaborative work. Experience gained together with indigenous partners from Latin America shows that the creation of new knowledge infrastructures requires a major rethink. It is not enough to open up the collections to the perspectives of others. Successful digital collaboration must reflect the heterogeneous infrastructures of knowledge of those involved.

Les plateformes numériques permettent d'ouvrir un accès plus large aux collections muséales et de faciliter leur exploration transculturelle. De 2015 à 2021, le projet « Partager des savoirs » du musée d'Ethnologie de Berlin s'est trouvé confronté aux défis inhérents à ce nouveau champ de travail collaboratif. L'expérience acquise au cours de collaborations avec des partenaires autochtones d'Amérique latine révèle que la création de nouvelles infrastructures de connaissance requiert, pour les musées et leur système d'archivage, d'importantes remises en question. Il ne suffit pas d'ouvrir les collections aux points de vue des autres. Le succès de la collaboration numérique suppose de réussir à y intégrer l'hétérogénéité des infrastructures de connaissance des différents participants.

ÍNDICE

Mots-clés: collections amazoniennes, décolonisation, retour numérique, infrastructure Palabras claves: colecciones amazónicas, descolonización, retorno digital, infraestructura Keywords: Amazonian collections, decolonization, digital return, infrastructure

\section{AUTORES}

\section{ANDREA SCHOLZ}

Museo Etnológico de los Museos Nacionales de Berlín/Fundación del Patrimonio Cultural Prusiano

\section{MIRIGÕ-DIANA GUZMÁN OCAMPO}

Escuela Normal Superior Indígena María Reina (Mitú/Colombia) 